\title{
Are social inequalities in acute myeloid leukemia survival explained by differences in treatment utilization? Results from a French longitudinal observational study among older patients
}

\author{
Eloïse Berger ${ }^{1 *}$ (D) Cyrille Delpierre ${ }^{1}$, Fabien Despas ${ }^{1,2}$, Sarah Bertoli ${ }^{3}$, Emilie Bérard ${ }^{1,4}$, Oriane Bombarde ${ }^{2}$,
}

Pierre Bories ${ }^{3,5}$, Audrey Sarry ${ }^{3}$, Guy Laurent ${ }^{1}$, Christian Récher ${ }^{3,6}$ and Sébastien Lamy ${ }^{1,2}$

\begin{abstract}
Background: Evidences support social inequalities in cancer survival. Studies on hematological malignancies, and more specifically Acute Myeloid Leukemia (AML), are sparser. Our study assessed: 1/ the influence of patients' socioeconomic position on survival, 2 / the role of treatment in this relationship, and $3 /$ the influence of patients' socioeconomic position on treatment utilization.

Methods: This prospective multicenter study includes all patients aged 60 and older, newly diagnosed with AML, excluding promyelocytic subtypes, between 1st January 2009 to 31st December 2014 in the South-West of France. Data came from medical files. Patients' socioeconomic position was measured by an ecological deprivation index, the European Deprivation Index. We studied first, patients' socioeconomic position influence on overall survival $(n=$ 592), second, on the use of intensive chemotherapy $(n=592)$, and third, on the use of low intensive treatment versus best supportive care among patients judged unfit for intensive chemotherapy $(n=405)$.

Results: We found an influence of patients' socioeconomic position on survival (highest versus lowest position $H_{R_{Q} 5}: 1.39[1.05 ; 1.87]$ that was downsized to become no more significant after adjustment for AML ontogeny (HR $R_{Q 5}$ : $1.31[0.97 ; 1.76]$ and cytogenetic prognosis $\left.\mathrm{HR}_{\mathrm{Q} 5}: 1.30[0.97 ; 1.75]\right)$. The treatment was strongly associated with survival. A lower proportion of intensive chemotherapy was observed among patients with lowest socioeconomic position $\left(\mathrm{OR}_{\mathrm{Q} 5}: 0.41[0.19 ; 0.90]\right)$ which did not persist after adjustment for AML ontogeny (OR $\left.\mathrm{Q}_{\mathrm{Q} 5}: 0.59[0.25 ; 1.40]\right)$. No such influence of patients' socioeconomic position was found on the treatment allocation among patients judged unfit for intensive chemotherapy.
\end{abstract}

Conclusions: Finally, these results suggest an indirect influence of patients' socioeconomic position on survival through AML initial presentation.

Keywords: Acute myeloid leukemia, Observational study, French European deprivation index, Cancer management and survival, Elderly patients

\footnotetext{
* Correspondence: eloise.berger@univ-tlse3.fr

${ }^{1}$ LEASP, UMR 1027, Equipe labellisée Ligue Nationale Contre le Cancer,

Faculté de médecine de Purpan, Inserm-Université Toulouse III Paul Sabatier,

37 allées Jules Guesde, 31000 Toulouse, France

Full list of author information is available at the end of the article
}

(c) The Author(s). 2019 Open Access This article is distributed under the terms of the Creative Commons Attribution 4.0 International License (http://creativecommons.org/licenses/by/4.0/), which permits unrestricted use, distribution, and

reproduction in any medium, provided you give appropriate credit to the original author(s) and the source, provide a link to the Creative Commons license, and indicate if changes were made. The Creative Commons Public Domain Dedication waiver (http://creativecommons.org/publicdomain/zero/1.0/) applies to the data made available in this article, unless otherwise stated. 


\section{Background}

Many studies support that social inequalities may exist at all steps of cancer care pathway, from the early stages of cancer development to survival [1-5]. Patients' socioeconomic position (SEP)-related differences in stage at diagnosis and access to treatment have been pointed out as the most important explanatory factors of social inequalities in mortality. However, results may vary depending on the healthcare system specificity as, for instance, people in a public tax-supported healthcare setting would be less exposed to financial barrier to care than in private funding healthcare settings $[1,6]$. In most cases, studies concerned solid tumors and very few papers have focused on hematological malignancies More specifically, studies dealing with the influence of SEP on acute myeloid leukemia (AML) care and outcome are sparser. In the USA, i.e. in a health system mainly based on private funding, ethnicity, insurance status, educational level, and income were found to affect overall survival [7-10], at least partially through SEPrelated inequality in treatment utilization, mainly access to intensive therapy and hematopoietic stem cell transplantation [7, 8, 10-13]. In Scandinavia, where healthcare services are mainly public or tax-supported, studies supported an association between overall survival and SEP measured by occupational class [14], and education level [15] although this relationship was not observed systematically. Regarding SEP-related differences in treatment utilization, results differed from those observed in private funding healthcare setting [15], with a lower use of intensive therapy in the lower educational level group but only among older AML patients. This indicates that, in addition to the healthcare system, the influence of patients' SEP on AML treatment and outcome may involve different mechanisms depending on patients' age. Incidence of AML increases sharply with age and standard care regimens for older AML patients are based primarily on three perspectives: (1) intensive chemotherapy, which are toxic but curative; (2) hypomethylating agents as semi-palliative but active approach and (3) best supportive care. To our knowledge, study assessing the influence of SEP on treatment utilization, especially among older patients, only focused on the use of intensive therapy. In response to this, the present study aims at studying: $1 /$ the influence of patients' SEP on survival, $2 /$ the role of treatment in this relationship, and $3 /$ the influence of patients' SEP on treatment utilization using a prospective AML database from the multicentric oncology network Onco-Occitanie in the Southwest of France. Here, SEP-related differences in the choice of treatment are assumed to be a potential explanatory mechanism of SEP-related differences in survival.

\section{Methods}

Study design

The IUCT-O AML study is a prospective longitudinal study including all patients treated for an AML in the Midi-Pyrénées region in South-West of France (about 2.8 million of inhabitants) [16]. Patients diagnosed with AML are referred by personal physicians, primary care centers or directly, in the Leukaemia unit of the Toulouse University Hospital. Data are centralized at the University Hospital and recorded each week according to guidelines from the oncology healthcare network of the Midi Pyrenees region (ONCOMIP) [17]. The IUCT$\mathrm{O}$ AML database is registered at the Commission Nationale de l'Informatique et des Libertés (CNIL) under $\mathrm{N}^{\circ} 1$, 778,920 . We included all patients aged 60 and older, newly diagnosed with an AML, excluding M3- subtypes, diagnosed between 1st January 2009 to 31st December 2014.

\section{Data collection}

Clinical data were collected from patients' medical files and certified by the Data Management Committee of the anonymized AML database of Toulouse University Hospital. Patients yielded written inform consent allowing the collection of personal clinical and biological data in an anonymized database. In accordance to the declaration of Helsinki, the study was reviewed and approved by the research ethics committee at Toulouse University Hospital. Regarding patients' outcome, we considered the time between diagnosis and death from all cause. Patients' were followed up to May 2017. The maximum length of follow-up was 6 years and 8 months and half of the sample was followed at least 4 months. Treatment were categorized as intensive chemotherapy (IC), low intensity therapy (LIT) and best supportive care (BSC). LIT and BSC were considered as non-intensive therapy. Intensive chemotherapy regimen as well as treatment with hypomethylating agents has been described elsewhere $[16,18]$. Due to the lack of individual SEP measures in medical record, we used an ecologicallevel measure of SEP to approach the patients' individual situation from the geographical coordinates of their addresses at the time of diagnosis. The French version of the European Deprivation Index (EDI) was developed to assess social deprivation [19], built from the Townsend's definition of deprivation as "a state of observable and demonstrable disadvantage relative to the local community or the wider society to which an individual, family or group belongs" [20]. For each address, we identify the geographical area of about 2000 inhabitants (IRIS) for which EDI was available. We consider the national quintile of EDI: living in the fifth quintile meant to live in an area belonging to 
the 20\% most deprived areas in France. In addition, patients' characteristics included age, sex, comorbidity assessed by the Charlson cormorbidity index ( $\mathrm{cci}=0$, 1 , or $\geq 2$ ) [21] and performance status. The disease characteristics included white blood cell count (sample distribution tercile), AML ontogeny, i.e. de novo vs. secondary AML (including post-myelodysplastic syndrome AML, post-chronic myelomonocytic AML, post-myeloproliferative disorder AML and therapy-related AML), and cytogenetic prognosis was defined according to the refined British MRC classification [22].

\section{Statistical analysis}

We use a theory-driven approach to study whether patients' SEP affect survival directly or through potential intermediate factors. In response to our two first objectives (step 1 analysis), we tested the influence of patients' SEP on overall survival (objective 1) and the effect of the adjustment for treatment on the SEP survival relationship (objective 2). We used Cox models with time-varying component for survival analyses to correct for non-proportional hazards. Then, we focused on the influence of patients' SEP on the treatment received. As previously suggested by Bories et al., we assumed induction treatment choice to be a 2 -steps process. First, the patients' fitness for IC is assessed (step 2 analysis). Then, among those judged unfit for IC, the fitness for LIT is assessed (step 3 analysis). Accordingly, we built a two-step analysis testing for SEP-related differences in $1 /$ receiving IC or not among all patients, and $2 /$ receiving LIT or BSC among patients judged unfit for IC. We built generalized linear models estimating the probability of receiving 1/ IC (versus LIT or BSC), and 2/ LIT (versus BSC) as a function of EDI quintile (ref: the less deprived quintile (quintile 1)). Covariates were entered in models, first alternatively, and then simultaneously to assess potential intermediate variables in the pathway linking patients' SEP to survival and treatment. All models were systematically adjusted for age, sex, and comorbidity. Potential confounders were identified from bivariate analyses as being associated with the outcomes, i.e. the death from all cause or the selected treatment. We fixed type I errors threshold to 0.2 and 0.05 for respectively bivariate and multivariable analyses. In sensitivity analysis we used multiple imputation methods for dealing missing data on both patients' SEP and confounders [23, 24]. Imputation models were based on the available information regarding patients' age, sex, performance status, AML ontogeny, level of white blood cells, and also the treatment received [25]. All analyses were done by using STATA release 14 (StataCorp LP, College Station, TX, USA).

\section{Results}

\section{Selection of the study population}

The flowchart is presented in Fig. 1. Among the 705 eligible patients, 113 were excluded due to missing data on treatment, SEP, or covariates. The resulting study sample included 592 patients. As shown in Table 1, compared to these patients, those excluded were significantly older, less often men, more often treated by LIT (especially by low dose cytarabine), with less favorable clinical characteristics at the exception of white blood cell count for which no statistically significant difference was found, and their patients' clinical characteristics were most often undefined. Excluded patients had also poorer overall survival (median survival $[95 \% \mathrm{CI}]$ in years $=0.18$ $[0.10 ; 0.42]$ versus $0.58[0.45,0.72]$ for included patients).

\section{Description of the study population}

From Table 1, IC, LIT and BSC represented respectively 32, 38 and $30 \%$ of the 592 patients included study sample. In total, $68 \%$ of the study sample $(n=405)$ did not receive IC. The distribution of patients between EDI levels was fairly balanced. Table 2 presented the distribution of patients' characteristics according to their socioeconomic position. In bivariate analyses (Additional files 1, 2 and 3: Tables S1 to S3), poorer overall survival was associated with non-intensive therapy, the highest level of social deprivation, advanced age, higher level of comorbidity, poorer performance status, higher level of WBC, secondary or undefinable AML ontogeny, and unfavorable or undefinable cytogenetic prognosis (Additional file 1: Table S1). Regarding the treatment, using IC or non-IC was associated with social deprivation index, sex, age, comorbidity, performance status, WBC count, AML ontogeny, and cytogenetic prognosis (Additional file 2: Table S2). Among patients judged as not fit for IC, using low IT or BSC was associated with sex, comorbidity, performance status, and WBC count (Additional file 3: Table S3).

\section{Influence of SEP on overall survival}

Table 3 presents the results from step 1 testing for the influence of patients' SEP on overall survival. As shown by model 1.0 results, compared to patients from the least deprived areas, those living in the most deprived areas had a higher risk of dying from all causes that was not explained by differences in age, sex or comorbidity. Models 1.1 to 1.5 showed that the influence of the lowest SEP on survival was downsized to become not statistically significant after adjustment for AML ontogeny, and cytogenetic prognosis. Conversely, this effect resisted to adjustment for performance status, WBC and treatment. In models 1.6 and 1.7, we did not find any persisting influence of patients' SEP on overall survival that was not explained by covariates presents in the models. 


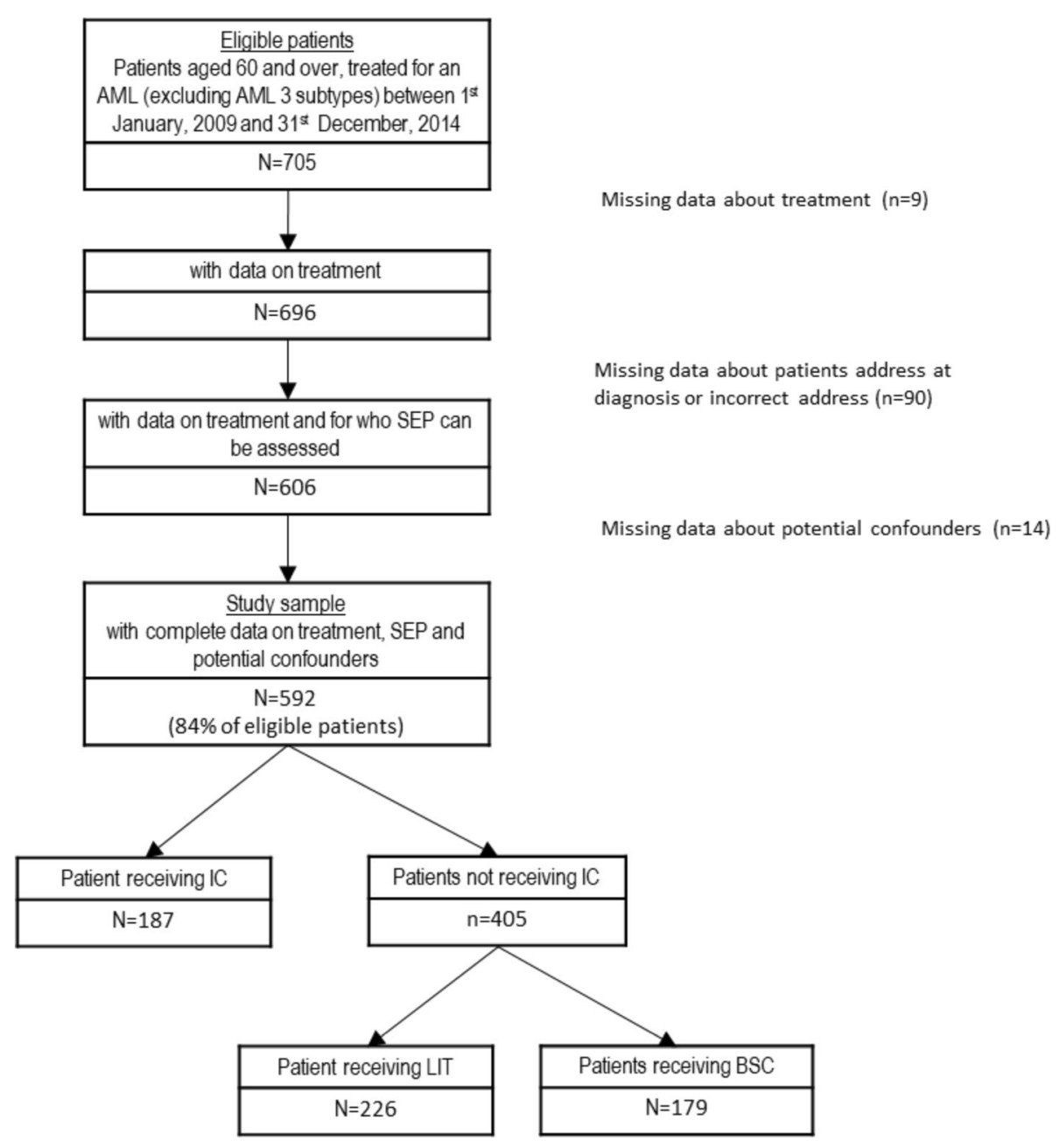

Fig. 1 flowchart

Regarding the other factors, results from models 1 showed that aging, poorer performance status levels, poor cytogenetic prognosis, and high values of WBC were associated with poorer survival. Results from the "time varying component" section indicates that the effect of WBC count on survival decreased with time from diagnosis.

\section{Influence of SEP on therapeutic strategies}

Table 4 presents the results from step 2 testing for the influence of patients' SEP on the probability of receiving or not IC. In model 2.0 patients with the lowest SEP had lower access to IC than those with the highest SEP. From the models 2.1 to 2.4, we observed that this association was downsized to become not statistically significant after adjustment for AML ontogeny, and cytogenetic prognosis but it was not affected by adjustment for performance status and WBC count. In model 2.5 results, patients' SEP had no more influence on the use of IC. Regarding the other factors, model 2.5 shows that the probability of receiving IC was lower among older patients, undefinable comorbidity level, poorer performance status, secondary (post-treatment or MDS) AML, and unfavorable cytogenetic prognosis. Conversely, higher level of white blood cell count was associated with higher probability of receiving IC.

Table 5 presents the results from step 3 testing for the influence of deprivation on the probability of receiving low intensive therapy or not, i.e. BSC, among patients judged unfit for IC $(n=405)$. Results from models 3.0 to 3.3 did not show any statistically significant influence of patients' SEP. Regarding the other factors, as expected, ageing, comorbidity, poorer performance status levels, and higher WBC count were associated with lower probability of receiving LIT.

\section{Sensitivity analyses}

In sensitivity analyses, we found the same pattern of results but with larger confidence intervals. The 
Table 1 Comparison between the excluded and the study samples characteristics (total $N=705$ )

\begin{tabular}{|c|c|c|c|c|c|c|}
\hline & & \multicolumn{2}{|c|}{$\begin{array}{l}\text { Excluded sample } \\
(n=113)\end{array}$} & \multicolumn{2}{|c|}{$\begin{array}{l}\text { Study sample } \\
(n=592)\end{array}$} & \multirow{2}{*}{$\begin{array}{l}\text { Test } \\
\text { comparing } \\
\text { study sample } \\
\text { with } \\
\text { excluded } \\
\text { sample } \\
\text { characteristics }\end{array}$} \\
\hline & & $\mathrm{N}$ & $\%$ or mean $(s d)$ & $\mathrm{N}$ & $\%$ or mean $(s d)$ & \\
\hline \multirow[t]{5}{*}{ Treatment (total $N=696$ ) } & Intensive chemotherapy & 7 & 7 & 187 & 32 & \multirow[t]{5}{*}{$p^{*}<0.001$} \\
\hline & Best supporting care & 81 & 12 & 179 & 30 & \\
\hline & Hypomethylating agents & 12 & 3 & 175 & 7 & \\
\hline & Aracytine low dose & 3 & 78 & 42 & 30 & \\
\hline & Others & 1 & 1 & 9 & 1 & \\
\hline \multicolumn{7}{|l|}{ Patient's characteristics } \\
\hline Age & Median (inter-quartile range) & 80 & 10 & 74 & 12 & $p^{\#}<0.001$ \\
\hline \multirow[t]{2}{*}{ Sex } & Men & 59 & 52 & 364 & 61 & \multirow[t]{2}{*}{$p^{\S}=0.065$} \\
\hline & Women & 54 & 48 & 228 & 39 & \\
\hline \multirow{5}{*}{$\begin{array}{l}\text { Patients' SEP (EDI quintile) } \\
\text { (total } N=613 \text { ) }\end{array}$} & Q1 - least deprived & 3 & 14 & 124 & 21 & \\
\hline & Q2 & 6 & 29 & 104 & 18 & \\
\hline & Q3 & 6 & 29 & 127 & 21 & \multirow[t]{3}{*}{$p^{*}=0.388$} \\
\hline & Q4 & 5 & 24 & 137 & 23 & \\
\hline & Q5 - most deprived & 1 & 5 & 100 & 17 & \\
\hline \multirow[t]{4}{*}{ Charlson comorbidity index } & 0 & 20 & 19 & 261 & 44 & \\
\hline & 1 & 11 & 10 & 122 & 21 & \multirow[t]{3}{*}{$p^{\S}=0.001$} \\
\hline & $2+$ & 12 & 11 & 104 & 18 & \\
\hline & Undefinable & 64 & 60 & 105 & 18 & \\
\hline \multirow[t]{4}{*}{ Performance status } & $0 / 1$ & 26 & 25 & 309 & 52 & \\
\hline & 2 & 13 & 13 & 91 & 15 & \multirow[t]{3}{*}{$p^{\S}=0.001$} \\
\hline & $3 / 4$ & 10 & 10 & 60 & 10 & \\
\hline & Undefinable & 55 & 53 & 132 & 22 & \\
\hline \multicolumn{7}{|l|}{ Tumor's characteristics } \\
\hline \multirow[t]{4}{*}{ White blood cell counts (tercile) (total $N=599$ ) } & Tercile 1 - low & 1 & 14 & 195 & 33 & \\
\hline & Tercile 2 - intermediate & 4 & 57 & 189 & 32 & \multirow[t]{3}{*}{$p^{*}=0.393$} \\
\hline & Tercile 3 - high & 2 & 29 & 193 & 33 & \\
\hline & Undefinable & 0 & 0 & 15 & 3 & \\
\hline \multirow[t]{3}{*}{ AML ontogeny (total $N=704$ ) } & AML de novo & 36 & 32 & 301 & 51 & \\
\hline & Secondary AML (post treatment / MDS) & 41 & 37 & 268 & 45 & \multirow[t]{2}{*}{$p^{\S}<0.001$} \\
\hline & Undefinable & 35 & 31 & 23 & 4 & \\
\hline \multirow[t]{3}{*}{ Cytogenetic initial prognosis } & Favorable/Intermediate & 50 & 44 & 343 & 58 & \multirow[t]{3}{*}{$p^{\S}<0.001$} \\
\hline & Unfavorable & 26 & 23 & 203 & 34 & \\
\hline & Undefinable & 37 & 33 & 46 & 8 & \\
\hline
\end{tabular}

p-value for Fisher test *, chi-square test $\S$, or Wilcoxon \#

detailed results are presented in Additional files 4, 5 and 6: Tables S4, S5 and S6.

\section{Discussion}

We found an association linking patients' SEP to overall survival that did not persist after adjustment for AML and patients' characteristics. As expected, the type of treatment was strongly associated with survival. However, its role as intermediate factor in the pathway linking patients' SEP to survival is not supported by our results. Indeed, we showed a statistically significant lower propensity of being treated using intensive chemotherapy among patients with lowest SEP but this did not persist after adjustment for AML ontogeny and cytogenetic 
Table 2 Distribution of the study sample characteristics by patients' socioeconomic position $(n=592)$

\begin{tabular}{|c|c|c|c|c|c|c|c|c|c|c|c|}
\hline & & \multicolumn{10}{|c|}{ Patients' SEP (EDI quintile) } \\
\hline & & \multicolumn{2}{|c|}{0 (least deprived) } & \multicolumn{2}{|l|}{1} & \multicolumn{2}{|l|}{2} & \multicolumn{2}{|l|}{3} & \multicolumn{2}{|c|}{4 (most deprived) } \\
\hline & & $\mathrm{n}$ & $\%$ & $\mathrm{n}$ & $\%$ & $\mathrm{n}$ & $\%$ & $\mathrm{n}$ & $\%$ & $n$ & $\%$ \\
\hline \multirow[t]{2}{*}{ Sex } & Men & 74 & 59.68 & 71 & 68.27 & 70 & 55.12 & 86 & 62.77 & 63 & 63.00 \\
\hline & Women & 50 & 40.32 & 33 & 31.73 & 57 & 44.88 & 51 & 37.23 & 37 & 37.00 \\
\hline \multicolumn{2}{|c|}{ Age (median (interquartile range)) } & 74 & 15.5 & 75 & 13.00 & 74 & 11.00 & 73 & 12.00 & 75 & 12.50 \\
\hline \multirow[t]{4}{*}{ Charlson comorbidity index } & 0 & 59 & 47.58 & 51 & 49.04 & 54 & 42.52 & 61 & 44.53 & 36 & 36.00 \\
\hline & 1 & 27 & 21.77 & 20 & 19.23 & 21 & 16.54 & 26 & 18.98 & 28 & 28.00 \\
\hline & $2+$ & 19 & 15.32 & 18 & 17.31 & 22 & 17.32 & 30 & 21.90 & 15 & 15.00 \\
\hline & Undefinable & 19 & 15.32 & 15 & 14.42 & 30 & 23.62 & 20 & 14.60 & 21 & 21.00 \\
\hline \multirow[t]{4}{*}{ Performance status } & $0 / 1$ & 70 & 56.45 & 59 & 56.73 & 67 & 52.76 & 72 & 52.55 & 41 & 41.00 \\
\hline & 2 & 14 & 11.29 & 18 & 17.31 & 15 & 11.81 & 23 & 16.79 & 21 & 21.00 \\
\hline & $3 / 4$ & 8 & 6.45 & 10 & 9.62 & 15 & 11.81 & 16 & 11.68 & 11 & 11.00 \\
\hline & Undefinable & 32 & 25.81 & 17 & 16.35 & 30 & 23.62 & 26 & 18.98 & 27 & 27.00 \\
\hline \multirow[t]{4}{*}{ White blood cell (terticle } & Low & 35 & 28.23 & 35 & 33.65 & 37 & 29.13 & 55 & 40.15 & 33 & 33.00 \\
\hline & Medium & 43 & 34.68 & 36 & 34.62 & 44 & 34.65 & 38 & 27.74 & 28 & 28.00 \\
\hline & High & 44 & 35.48 & 33 & 31.73 & 41 & 32.28 & 41 & 29.93 & 34 & 34.00 \\
\hline & Undefinable & 2 & 1.61 & 0 & 0.00 & 5 & 3.94 & 3 & 2.19 & 5 & 5.00 \\
\hline \multirow[t]{3}{*}{ AML ontogeny } & AML de novo & 74 & 59.68 & 51 & 49.04 & 63 & 49.61 & 73 & 53.28 & 40 & 40.00 \\
\hline & Secondary AML (post treatment / MDS) & 46 & 37.10 & 49 & 47.12 & 59 & 46.46 & 60 & 43.80 & 54 & 54.00 \\
\hline & Undefinable & 4 & 3.23 & 4 & 3.85 & 5 & 3.94 & 4 & 2.92 & 6 & 6.00 \\
\hline \multirow[t]{3}{*}{ Cytogenetic prognosis } & Favorable/Intermediate & 77 & 62.10 & 54 & 51.92 & 76 & 59.84 & 84 & 61.31 & 52 & 52.00 \\
\hline & Unfavorable & 38 & 30.65 & 44 & 42.31 & 39 & 30.71 & 42 & 30.66 & 40 & 40.00 \\
\hline & Undefinable & 9 & 7.26 & 6 & 5.77 & 12 & 9.45 & 11 & 8.03 & 8 & 8.00 \\
\hline \multirow[t]{3}{*}{ Treatment } & Intensive chemotherapy & 50 & 40.32 & 28 & 26.92 & 42 & 33.07 & 44 & 32.12 & 23 & 23.00 \\
\hline & Low intensive therapy & 40 & 32.26 & 43 & 41.35 & 45 & 35.43 & 57 & 41.61 & 41 & 41.00 \\
\hline & Best supportive care & 34 & 27.42 & 33 & 31.73 & 40 & 31.50 & 36 & 26.28 & 36 & 36.00 \\
\hline
\end{tabular}

prognosis. This may indicate that, patients' and AML initial characteristics being equal, patients' SEP do not influence the utilization of intensive chemotherapy. However, we cannot exclude an indirect influence of patients' SEP on the utilization of intensive chemotherapy and survival through SEP-related differences in AML initial presentation and cytogenetic prognosis. No such influence of patients' SEP was found on the propension of having low intensive therapy or BSC among patients judged unfit for IC.

This study aimed at testing for SEP-related differences in cancer management and outcome among old patients (60 years and over) in a setting of a national tax-supported healthcare system. We used data from an ongoing prospective observational cohort including all patients newly diagnosed for an AML in the South-West of France since 2007. In France, the healthcare organization is centralized and relayed at the regional level by Regional Health Agency. Many efforts were done for standardizing and harmonizing cancer management, notably with the implementation of the national cancer plans which aimed, amongst others, at developing regional cancer coordination centers responsible of the holding of multidisciplinary team meeting (MTM) for the first plan (2003-2007) and the reduction of social and territorial inequalities in cancer management for the second and third plans (2009-2013/ 2014-2019). One role of the regional cancer coordination centers is notably to ensure the diffusion of clinical guidelines throughout all the region centers. Thus, despite the lack of data for the whole national territory, we assumed that it is unlikely to affect the generalization of our results. However, our results showed that patients excluded from the study were not different regarding SEP but had less often intensive treatment, less favorable clinical characteristics and poorer survival. Thus, we may have underestimated the influence of SEP on both treatment and survival. Lastly, data were collected from medical files which did not contain any information on individual SEP like patients' occupation or education level or income. Therefore, we used an 
Table 3 Step 1. Survival in association with patients' SEP adjusted for treatment, patients' and disease characteristics

\begin{tabular}{|c|c|c|c|c|c|c|c|c|c|c|c|c|c|c|c|c|c|}
\hline \multirow[t]{3}{*}{ Main components } & & \multirow{2}{*}{\multicolumn{2}{|c|}{ Model 1.0 (M1.0) }} & \multirow{2}{*}{\multicolumn{2}{|c|}{$\begin{array}{l}\text { Model } 1.1 \\
\text { M1.0+ perf. } \\
\text { Status }\end{array}$}} & \multirow{2}{*}{\multicolumn{2}{|c|}{ 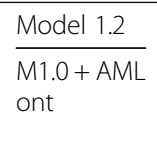 }} & \multirow{2}{*}{\multicolumn{2}{|c|}{$\begin{array}{l}\text { Model } 1.3 \\
\text { M1.0+ } \\
\text { WBC. }\end{array}$}} & \multirow{2}{*}{\multicolumn{2}{|c|}{$\begin{array}{l}\text { Model } 1.4 \\
\text { M1.0+ } \\
\text { cyto. } \\
\text { Progn. }\end{array}$}} & \multirow{2}{*}{\multicolumn{2}{|c|}{$\begin{array}{l}\text { Model } 1.5 \\
\text { M1.0+ } \\
\text { treatment }\end{array}$}} & \multirow{2}{*}{\multicolumn{2}{|c|}{$\begin{array}{l}\text { Model } 1.6 \\
\text { All but } \\
\text { treatment }\end{array}$}} & \multirow{2}{*}{\multicolumn{2}{|c|}{$\begin{array}{l}\text { M } 1.7 \\
\text { Fully } \\
\text { adjusted }\end{array}$}} \\
\hline & & & & & & & & & & & & & & & & & \\
\hline & & $\overline{\mathrm{HR}}$ & $\begin{array}{l}95 \% \\
\mathrm{Cl}]\end{array}$ & $\mathrm{HR}$ & $\begin{array}{l}{[95 \%} \\
\mathrm{Cl}]\end{array}$ & $\overline{\mathrm{HR}}$ & $\begin{array}{l}{[95 \%} \\
\mathrm{CI}]\end{array}$ & $\mathrm{HR}$ & $\begin{array}{l}{[95 \%} \\
\mathrm{Cl}]\end{array}$ & $\overline{\mathrm{HR}}$ & $\begin{array}{l}{[95 \%} \\
\mathrm{Cl}]\end{array}$ & $\overline{\mathrm{HR}}$ & $\begin{array}{l}95 \% \\
\mathrm{Cl}]\end{array}$ & $\overline{\mathrm{HR}}$ & $\begin{array}{l}{[95 \%} \\
\mathrm{Cl}]\end{array}$ & $\overline{\mathrm{HR}}$ & $\begin{array}{l}{[95 \%} \\
\mathrm{Cl}]\end{array}$ \\
\hline Age & & 1.04 & {$[1.03 ; 1.05]$} & 1.03 & $\begin{array}{l}1.02 ; \\
1.05]\end{array}$ & 1.04 & $\begin{array}{l}{[1.03 ;} \\
1.05]\end{array}$ & 1.04 & $\begin{array}{l}{[1.03 ;} \\
1.05]\end{array}$ & 1.04 & $\begin{array}{l}{[1.02 ;} \\
1.05]\end{array}$ & 1.01 & $\begin{array}{l}{[1.00 ;} \\
1.03]\end{array}$ & 1.03 & $\begin{array}{l}1.02 ; \\
1.05]\end{array}$ & 1.02 & $\begin{array}{l}\text { [1.00; } \\
1.03]\end{array}$ \\
\hline \multirow[t]{2}{*}{ Sex } & Men & ref & & ref & & ref & & ref & & & & ref & & Ref & & ref & \\
\hline & Women & 0.88 & {$[0.73 ; 1.07]$} & 0.86 & $\begin{array}{l}{[0.71 ;} \\
1.04]\end{array}$ & 0.87 & $\begin{array}{l}{[0.72 ;} \\
1.05]\end{array}$ & 0.89 & $\begin{array}{l}{[0.74 ;} \\
1.08]\end{array}$ & 0.84 & $\begin{array}{l}{[0.69 ;} \\
1.01]\end{array}$ & 0.87 & $\begin{array}{l}{[0.72 ;} \\
1.05]\end{array}$ & 0.81 & $\begin{array}{l}{[0.67 ;} \\
0.98]\end{array}$ & 0.82 & $\begin{array}{l}{[0.68 ;} \\
1.00]\end{array}$ \\
\hline Patients' SEP (quintile & Q1 - least & ref & & Ref & & Ref & & Ref & & & & Ref & & ref & & Ref & \\
\hline & Q2 & 1.14 & {$[0.85 ; 1.53]$} & 1.11 & $\begin{array}{l}{[0.83 ;} \\
1.50]\end{array}$ & 1.08 & $\begin{array}{l}{[0.80} \\
1.45]\end{array}$ & 1.17 & $\begin{array}{l}{[0.87 ;} \\
1.35]\end{array}$ & 1.06 & $\begin{array}{l}{[0.79 ;} \\
1.42]\end{array}$ & 1 & $\begin{array}{l}{[0.84 ;} \\
1.50]\end{array}$ & 1.01 & $\begin{array}{l}{[0.75 ;} \\
1.37]\end{array}$ & 0.96 & $\begin{array}{l}{[0.71 ;} \\
1.29]\end{array}$ \\
\hline & Q3 & 0.89 & {$[0.67 ; 1.18]$} & 0.85 & $\begin{array}{l}{[0.64 ;} \\
1.13]\end{array}$ & 0.83 & $\begin{array}{l}{[0.62 ;} \\
1.11]\end{array}$ & 0.88 & $\begin{array}{l}{[0.66 ;} \\
1.17]\end{array}$ & 0.89 & $\begin{array}{l}{[0.67 ;} \\
1.18]\end{array}$ & 0.84 & $\begin{array}{l}{[0.69 ;} \\
1.22]\end{array}$ & 0.80 & $\begin{array}{l}{[0.60 ;} \\
1.07]\end{array}$ & 0.78 & $\begin{array}{l}{[0.58 ;} \\
1.04]\end{array}$ \\
\hline & Q4 & 1.07 & {$[0.82 ; 1.40]$} & 1.02 & $\begin{array}{l}{[0.78 ;} \\
1.33]\end{array}$ & 1.03 & $\begin{array}{l}{[0.79 ;} \\
1.35]\end{array}$ & 1.07 & $\begin{array}{l}{[0.82 ;} \\
1.40]\end{array}$ & 1.06 & $\begin{array}{l}{[0.81 ;} \\
1.38]\end{array}$ & 1 & $\begin{array}{l}{[0.82 ;} \\
1.40]\end{array}$ & 0.99 & $\begin{array}{l}{[0.75 ;} \\
1.30]\end{array}$ & 0.94 & $\begin{array}{l}{[0.71 ;} \\
1.24]\end{array}$ \\
\hline & Q5 - most & 1.39 & {$[1.04 ; 1.87]$} & 1.37 & $\begin{array}{l}{[1.02 ;} \\
1.84]\end{array}$ & 1.31 & $\begin{array}{l}{[0.97 ;} \\
1.76]\end{array}$ & 1.47 & $\begin{array}{l}{[1.10 ;} \\
1.97]\end{array}$ & 1.30 & $\begin{array}{l}{[0.97 ;} \\
1.75]\end{array}$ & 1.35 & $\begin{array}{l}{[1.12 ;} \\
2.01]\end{array}$ & 1.28 & $\begin{array}{l}{[0.95 ;} \\
1.73]\end{array}$ & 1.29 & $\begin{array}{l}{[0.95 ;} \\
1.74]\end{array}$ \\
\hline Charlson comorbidity & 0 & ref & & ref & & ref & & ref & & & & ref & & ref & & ref & \\
\hline & 1 & 1.1 & {$[0.86 ; 1.41]$} & 1.02 & $\begin{array}{l}{[0.80 ;} \\
1.31]\end{array}$ & 1.08 & $\begin{array}{l}{[0.85 ;} \\
1.38]\end{array}$ & 1.15 & $\begin{array}{l}{[0.90 ;} \\
1.47]\end{array}$ & 1.09 & $\begin{array}{l}{[0.86 ;} \\
1.40]\end{array}$ & 1.01 & $\begin{array}{l}{[0.79 ;} \\
1.29]\end{array}$ & 1.07 & $\begin{array}{l}{[0.83 ;} \\
1.37]\end{array}$ & 1.00 & $\begin{array}{l}{[0.78 ;} \\
1.28]\end{array}$ \\
\hline & $2+$ & 1.29 & {$[1.01 ; 1.66]$} & 1.18 & $\begin{array}{l}{[0.92 ;} \\
1.52]\end{array}$ & 1.21 & $\begin{array}{l}{[0.94 ;} \\
1.56]\end{array}$ & 1.3 & $\begin{array}{l}{[1.01 ;} \\
1.66]\end{array}$ & 1.35 & $\begin{array}{l}{[1.06 ;} \\
1.73]\end{array}$ & 1.13 & $\begin{array}{l}{[0.88 ;} \\
1.45]\end{array}$ & 1.19 & $\begin{array}{l}{[0.92 ;} \\
1.54]\end{array}$ & 1.08 & $\begin{array}{l}{[0.83 ;} \\
1.41]\end{array}$ \\
\hline & Undefined & 2.1 & {$[1.60 ; 2.76]$} & 1.89 & $\begin{array}{l}{[1.39 ;} \\
2.56]\end{array}$ & 1.88 & $\begin{array}{l}{[1.40 ;} \\
2.53]\end{array}$ & 2.06 & $\begin{array}{l}{[1.56 ;} \\
2.73]\end{array}$ & 1.97 & $\begin{array}{l}{[1.49 ;} \\
2.61]\end{array}$ & 1.29 & $\begin{array}{l}{[0.96 ;} \\
1.74]\end{array}$ & 1.66 & $\begin{array}{l}{[1.20 ;} \\
2.31]\end{array}$ & 1.25 & $\begin{array}{l}{[0.89 ;} \\
1.75]\end{array}$ \\
\hline Performance status & $0 / 1$ & & & ref & & & & & & & & & & Ref & & ref & \\
\hline & 2 & & & 1.51 & $\begin{array}{l}{[1.16 ;} \\
1.97]\end{array}$ & & & & & & & & & 1.49 & $\begin{array}{l}{[1.15 ;} \\
1.95]\end{array}$ & 1.50 & $\begin{array}{l}\text { [1.15; } \\
1.95]\end{array}$ \\
\hline & $3 / 4$ & & & 2.36 & $\begin{array}{l}{[1.70 ;} \\
3.28]\end{array}$ & & & & & & & & & 1.90 & $\begin{array}{l}{[1.34 ;} \\
2.68]\end{array}$ & 1.72 & $\begin{array}{l}{[1.22 ;} \\
2.42]\end{array}$ \\
\hline & Undefined & & & 1.75 & $\begin{array}{l}{[1.24 ;} \\
2.48]\end{array}$ & & & & & & & & & 1.52 & $\begin{array}{l}{[1.04 ;} \\
2.20]\end{array}$ & 1.29 & $\begin{array}{l}{[0.88 ;} \\
1.88]\end{array}$ \\
\hline AML ontogeny & $\begin{array}{l}\text { AML de } \\
\text { novo }\end{array}$ & & & & & ref & & & & & & & & Ref & & ref & \\
\hline & $\begin{array}{l}\text { Secondary A } \\
\text { MDS) }\end{array}$ & ML (pc & pst treatment & & & 1.25 & $\begin{array}{l}{[1.03 ;} \\
1.52]\end{array}$ & & & & & & & 1.21 & $\begin{array}{l}{[0.99 ;} \\
1.48]\end{array}$ & 1.12 & $\begin{array}{l}{[0.91 ;} \\
1.38]\end{array}$ \\
\hline & Undefined & & & & & 1.56 & $\begin{array}{l}{[0.90 ;} \\
2.72]\end{array}$ & & & & & & & 1.60 & $\begin{array}{l}{[0.90 ;} \\
2.83]\end{array}$ & 1.52 & $\begin{array}{l}{[0.86 ;} \\
2.70]\end{array}$ \\
\hline $\begin{array}{l}\text { White blood cell } \\
\text { (WBC) counts (tercile) }\end{array}$ & $\begin{array}{l}\text { Tercile } 1 \text { - } \\
\text { low }\end{array}$ & & & & & & & ref & & & & & & & & Ref & \\
\hline & Terticle 2 - i & terme & ediate & & & & & 1.42 & $\begin{array}{l}{[1.10 ;} \\
1.83]\end{array}$ & & & & & 1.38 & $\begin{array}{l}{[1.06 ;} \\
1.79]\end{array}$ & 1.34 & $\begin{array}{l}{[1.04 ;} \\
1.74]\end{array}$ \\
\hline & Terticle $3-r$ & igh & & & & & & 2.24 & $\begin{array}{l}{[1.66 ;} \\
3.01]\end{array}$ & & & & & 2.16 & $\begin{array}{l}{[1.59 ;} \\
2.93]\end{array}$ & 2.36 & $\begin{array}{l}{[1.74 ;} \\
3.20]\end{array}$ \\
\hline & Undefined & & & & & & & 2.9 & $\begin{array}{l}{[1.50 ;} \\
5.60]\end{array}$ & & & & & 2.28 & $\begin{array}{l}{[1.14 ;} \\
4.56]\end{array}$ & 2.10 & $\begin{array}{l}{[1.05 ;} \\
4.21]\end{array}$ \\
\hline $\begin{array}{l}\text { Cytogenetic } \\
\text { prognosis }\end{array}$ & $\begin{array}{l}\text { Favorable/ } \\
\text { Intermediate }\end{array}$ & & & & & & & & & & & & & & & & \\
\hline & Unfavorable & & & & & & & & & 2.00 & $\begin{array}{l}{[1.64 ;} \\
2.43]\end{array}$ & & & 2.01 & $\begin{array}{l}{[1.64 ;} \\
2.46]\end{array}$ & 1.72 & $\begin{array}{l}{[1.38 ;} \\
2.13]\end{array}$ \\
\hline & Undefinable & & & & & & & & & 1.99 & $\begin{array}{l}{[1.34 ;} \\
2.96]\end{array}$ & & & 1.88 & $\begin{array}{l}{[1.24 ;} \\
2.86]\end{array}$ & 1.44 & $\begin{array}{l}{[0.95 ;} \\
2.18]\end{array}$ \\
\hline
\end{tabular}


Table 3 Step 1. Survival in association with patients' SEP adjusted for treatment, patients' and disease characteristics (Continued)

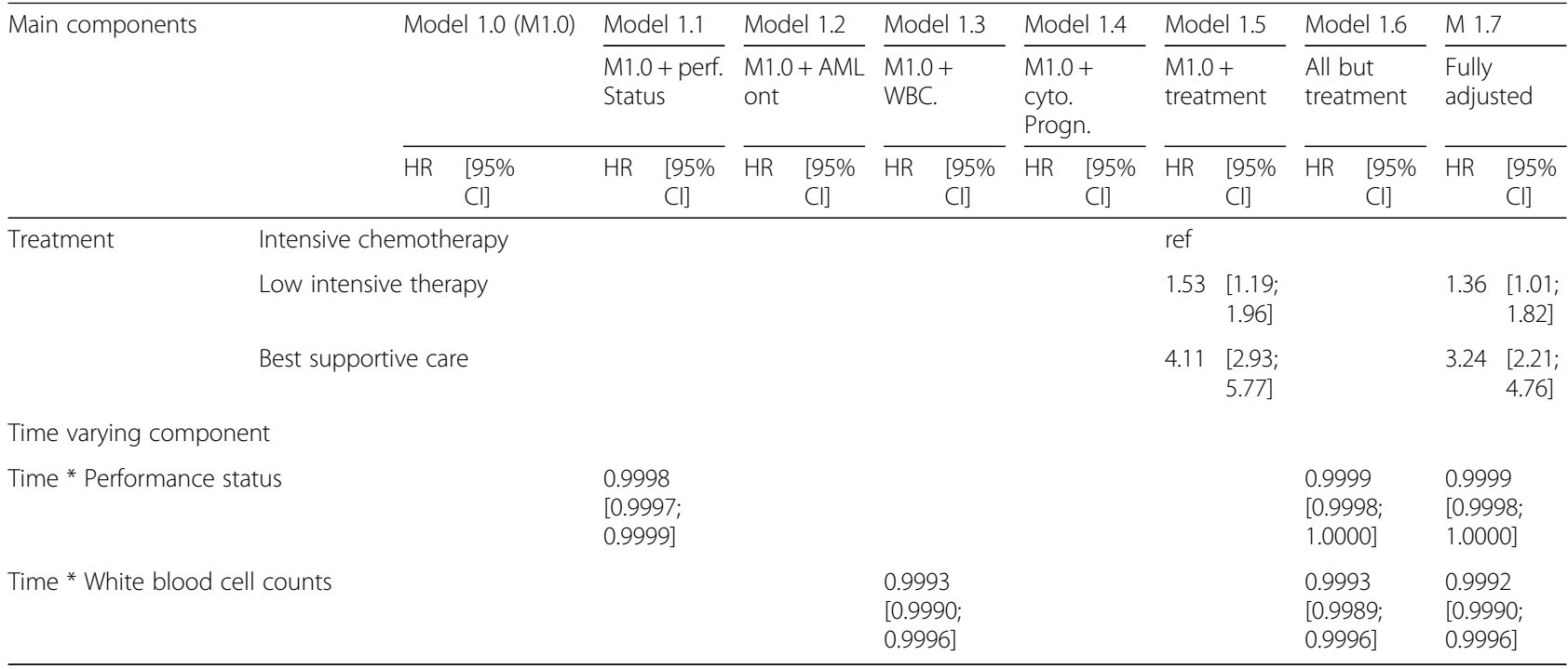

Adjusted hazard ratios [95\% Confidence Intervals] of overall mortality from Adjusted Cox proportional hazards model with time dependent variables ( $n=592$ )

ecological deprivation index to approach individual SEP despite the exposure to potential ecological fallacy. Indeed, as we attributed to patients the deprivation level of their living area to approach their individual SEP, it is possible that this measure hides some contextual dimension, like for instance environmental exposures. However, this is lessened as we used the French European Deprivation Index (EDI) at the smallest geographical area (the IRIS corresponding to approximately 2000 individuals) for which census data of the French population are available. The EDI has been previously used as patients' individual SEP proxy in studies dealing with social inequalities in cancer incidence [26], management [27] and outcome [28]. Moreover, a study published in early 2017 compared several deprivation indexes including the European Deprivation index (EDI), all aggregated at the IRIS level, and showed that the EDI was quite good "proxies" for individual deprivation (Area Under the Curve close to 0.7) [29].

To our knowledge, we found only two studies addressing SEP-related differences in AML management or outcome in a tax-supported healthcare setting. Regarding survival, our results cannot be compared to Kristinsson et al.'s [14] which concerns all AML patients without age restriction. In addition, we cannot compare our results to Østgård et al.'s study as they assessed SEP influence on survival only among patients selected for intensive chemotherapy [15]. In our study, we did not find any independent effect of patients' SEP after adjustment for both patients' and tumor's characteristics among patients aged of at least 60 years. More specifically, we found a SEP influence on survival that persisted in model adjusted for performance status, and WBC. This influence was reduced after adjustment for treatment and was downsized to become no more significant with adjustment for AML ontogeny, and cytogenetic prognosis. This suggested an indirect influence of SEP on survival through initial SEP-related differences in AML presentation even if we could not exclude, regarding to the slightly attenuation of the effect size, that the insignificant effect was due to lack of statistical power. When we consider the treatment utilization, the focus on tax-supported healthcare setting limits theoretically the effect of financial barrier to access to care. Østgård and colleagues' study supported the association between access to intensive therapy and education, as a proxy of SEP, among all patients as well as patients older than 60 . In addition, they found an independent effect of education after controlling for occupation, marital status and income on intensive treatment among older patients. No associations with income were found [15]. In our study, we found a lower access to intensive therapy among patients with the lowest SEP which persisted in model adjusted for performance status and WBC count but was downsized to become no more significant when accounting for AML ontogeny, and cytogenetic. This reinforced the role of the AML initial presentation in the SEP-survival association discussed above. Among patients who were judged unfit for intensive therapy, we found no more influence of patients' SEP. Finally compared to Østgård and colleagues' study, we did not show any independent persisting influence of SEP on survival and treatment allocation. This may indicate that, in our study region, patients' and AML initial characteristics being equal, patients' SEP do not influence the way AML is 


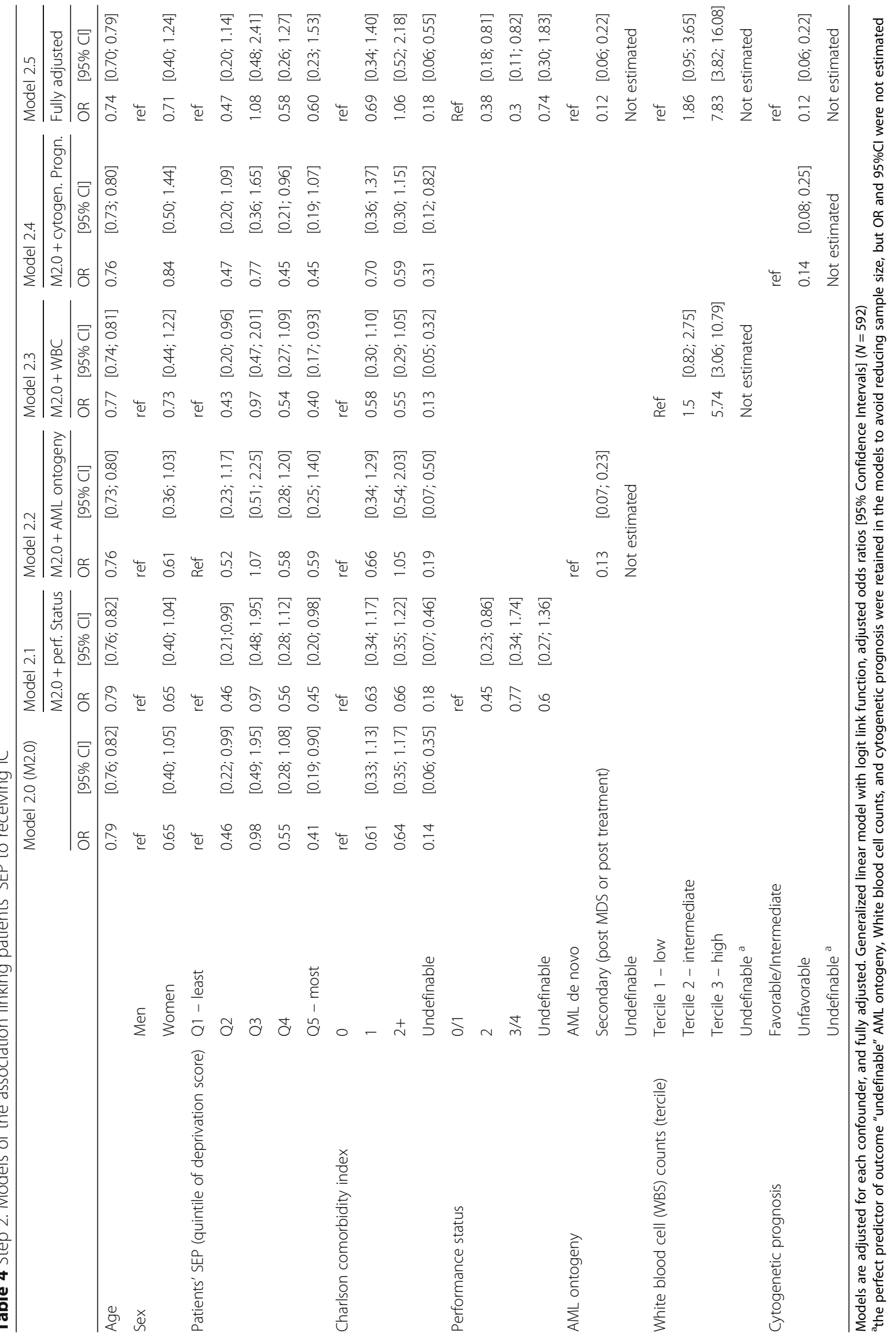


Table 5 Step 3. Adjusted models of the association linking patients' SEP to receiving non-intensive therapy $(n=405)$

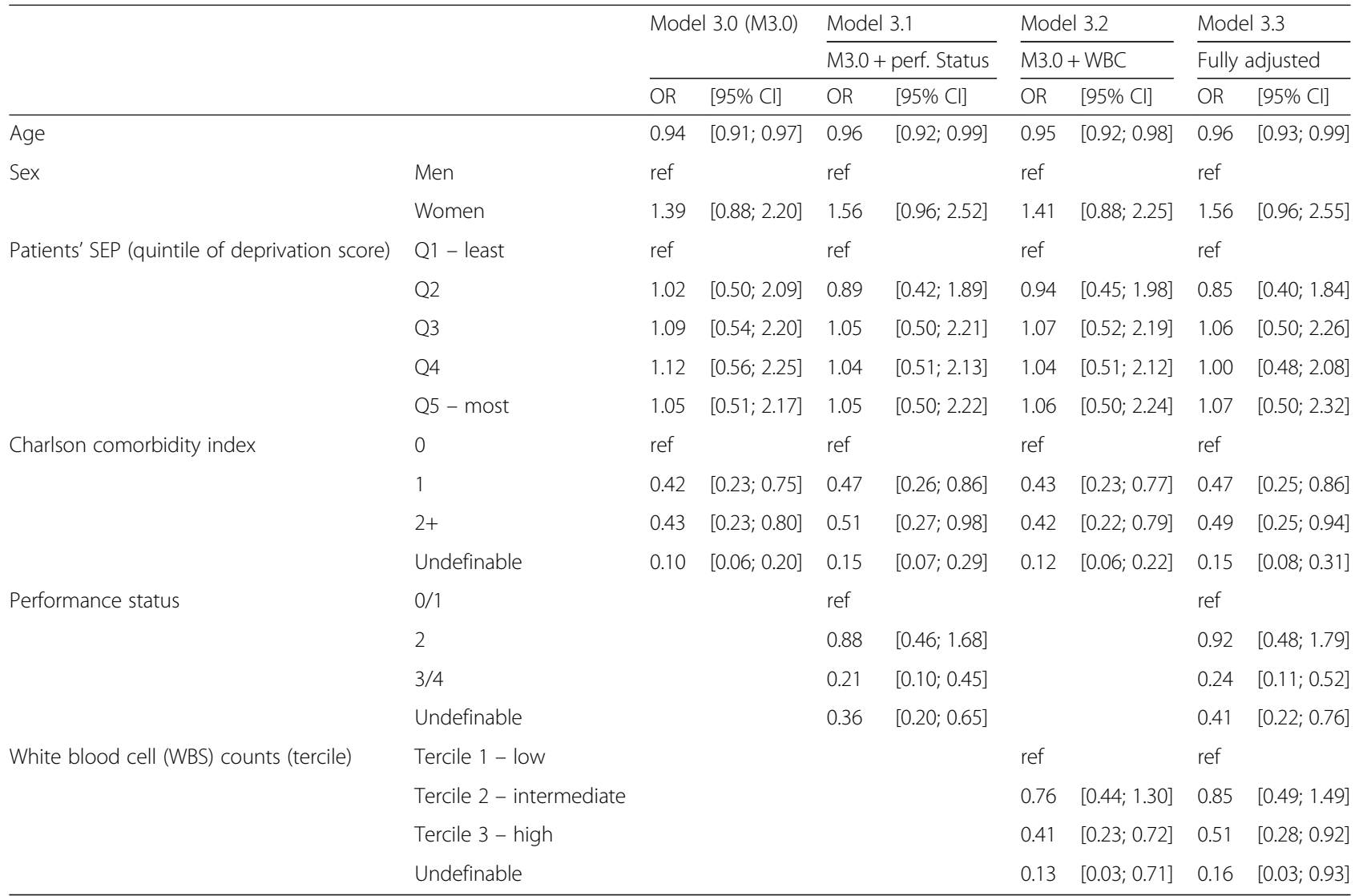

Population is selected among those who were not considered for intensive chemotherapy. Generalized linear model with logit link function, adjusted odds ratios [95\% Confidence Intervals]

treated nor its outcome. An indirect influence of patients' SEP on the utilization of intensive chemotherapy and survival is more likely through SEP-related differences in AML initial presentation and cytogenetic prognosis. Compared to Østgård and colleagues' study, the absence of persisting influence of SEP in our study may derive, at least partially, from differences in the study design as their study was based on populational registry whereas ours included patients from their entrance into the healthcare system. However, this also illustrates the variability of the mechanisms linking patients' SEP to survival trough, for instance, differences in management or in initial presentation depending potentially to various SEP dimensions.

\section{Conclusions}

The hypothesis of an indirect influence of SEP on survival through SEP-related differences in treatment utilization is not supported by our results, at least for the initial treatment. Adjusting survival model for treatment did not neutralize the SEP influence which seems rather to derive from SEP-related difference in AML ontogeny and cytogenetic prognosis. It therefore appears necessary to continue the investigation beyond the limits of treatment initiation and survival to identify at which points in the course of treatment, factors that might be considered as clinically irrelevant may be involved in the patient care trajectory. Especially further analyses are needed to test formally the assumption of an indirect influence of patients' SEP on survival through AML initial presentation and cytogenetic prognosis.

\section{Additional files}

Additional file 1: Table S1. Bivariate associations between covariates and overall survival. (DOCX $17 \mathrm{~kb}$ )

Additional file 2: Table S2. Bivariate associations between covariates and treatment selection in terms of intensive chemotherapy or not. (DOCX $15 \mathrm{~kb}$ )

Additional file 3: Table S3. Bivariate associations between covariates and treatment selection in terms of Low intensive chemotherapy or BSC. (DOCX $15 \mathrm{~kb}$ )

Additional file 4: Table S4. Step 1 sensitivity analysis. Survival in association with patients' SEP adjusted for treatment, patients' and disease characteristics. Adjusted hazard ratios [95\% Confidence Intervals] of overall mortality from Adjusted Cox proportional hazards model with time dependent variables $(n=684)$. (DOCX $23 \mathrm{~kb})$

Additional file 5: Table S5. Step 2 Sensitivity analysis. Models of the association linking patients' SEP to receiving Intensive Chemotherapy 
adjusted for each confounder, and fully adjusted. Generalized linear model with logit link function, adjusted odds ratios [95\% Confidence Intervals], with missing data treated by multiple imputation $(n=685)$. (DOCX $20 \mathrm{~kb}$ )

Additional file 6: Table S6. Step 3 Sensitivity analysis. Adjusted models of the association linking patients' SEP to receiving non-intensive therapy among those who were not considered for intensive chemotherapy $(n=$ 498). Generalized linear model with logit link function, adjusted odds ratios [95\% Confidence Intervals] after treating missing data using multiple imputation. (DOCX $17 \mathrm{~kb})$

\section{Abbreviations}

AML: Acute myeloid leukemia; BSC: Best supportive care; EDI: European deprivation index; IC: Intensive chemotherapy; LIT: Low intensive therapy; SEP: Socioeconomic position

\section{Acknowledgments}

The authors wish to thank the members of the CAPTOR WP3 project. This work was supported by La Ligue nationale contre le cancer [Equipe Labellisée LIGUE 2017/CD]). EB is supported by the Institut National du Cancer [SHSESP 2017-130 to CD].

\section{Authors' contributions}

$E B, C D, F B, P B, A S, C R, O B, G L$ and $S L$ designed research; $E B^{*}$ (EB* for Emilie Bérard), and $A S, O B, S B$ and $P B$ collected and controlled the data; $E B, C D$, $P B, S L, C R$ and $F D$ analyzed the data; $E B, C D, F D, S B, E B^{*}, P B, A S, G L, C R$ and $S L$ discussed the results and $E B, F D, P B, C D, C R$, and $S L$ wrote the paper. All authors reviewed and commented on the manuscript. All authors read and approved the final manuscript.

\section{Funding}

This work was supported by the Institut de Recherche en Santé Publique [SSC201504 to C.R.]. In addition, the French national Research Agency supported the early development of this study [ANR-11-PHUC-001 to G.L.]. The funding bodies did not have any role in the design of the study and collection, analysis and interpretation of data and in writing of the manuscript.

\section{Availability of data and materials}

The datasets used and/or analysed during the current study are available from the corresponding author on reasonable request.

\section{Ethics approval and consent to participate}

Patients yielded written inform consent allowing the collection of personal clinical and biological data in an anonymized database in accordance to the declaration of Helsinki. The study was reviewed and approved by the research ethics committee at Toulouse University Hospital. The IUCT-O AML database is registered at the Commission Nationale de l'Informatique et des Libertés (CNIL) under N¹778920.

\section{Consent for publication}

Not applicable.

\section{Competing interests}

The authors declare that they have no competing interests.

\section{Author details}

'LEASP, UMR 1027, Equipe labellisée Ligue Nationale Contre le Cancer, Faculté de médecine de Purpan, Inserm-Université Toulouse III Paul Sabatier, 37 allées Jules Guesde, 31000 Toulouse, France. ${ }^{2}$ Service de Pharmacologie Clinique, CHU de Toulouse, Toulouse, France. ${ }^{3}$ Service d'hématologie, Institut Universitaire du Cancer de Toulouse - Oncopôle, CHU de Toulouse, Toulouse, France. ${ }^{4}$ Service d'Epidemiologie, $\mathrm{CHU}$ de Toulouse, Toulouse, France. ${ }^{5}$ Réseau régional de cancérologie Onco-Occitanie, Institut Universitaire du Cancer de Toulouse Oncopôle, Toulouse, France. ${ }^{6}$ Centre de Recherche en Cancérologie de Toulouse UMR 1037 Inserm / ERL5294 CNRS, University of Toulouse 3 Paul Sabatier, Toulouse, France.
Received: 28 May 2019 Accepted: 26 August 2019

Published online: 05 September 2019

\section{References}

1. Woods LM, Rachet B, Coleman MP. Origins of socio-economic inequalities in cancer survival: a review. Ann Oncol. 2006;17(1):5-19.

2. Forrest LF, Sowden S, Rubin G, White M, Adams J. Socio-economic inequalities in stage at diagnosis, and in time intervals on the lung cancer pathway from first symptom to treatment: systematic review and metaanalysis. Thorax. 2017;72(5):430-6.

3. Klein J, von dem Knesebeck $\mathrm{O}$. Socioeconomic inequalities in prostate cancer survival: a review of the evidence and explanatory factors. Soc Sci Med. 2015;142:9-18.

4. Dialla PO, Arveux P, Ouedraogo S, Pornet C, Bertaut A, Roignot P, et al. Agerelated socio-economic and geographic disparities in breast cancer stage at diagnosis: a population-based study. Eur J Pub Health. 2015;25(6):966-72.

5. Barclay KL, Goh PJ, Jackson TJ. Socio-economic disadvantage and demographics as factors in stage of colorectal cancer presentation and survival. ANZ J Surg. 2015;85(3):135-9.

6. Quaglia A, Lillini R, Mamo C, Ivaldi E, Vercelli M. Socio-economic inequalities: a review of methodological issues and the relationships with cancer survival. Crit Rev Oncol Hematol. 2013;85(3):266-77.

7. Bierenbaum J, Davidoff AJ, Ning Y, Tidwell ML, Gojo I, Baer MR. Racial differences in presentation, referral and treatment patterns and survival in adult patients with acute myeloid leukemia: a single-institution experience. Leuk Res. 2012;36(2):140-5.

8. Patel MI, Ma Y, Mitchell B, Rhoads KF. How do differences in treatment impact racial and ethnic disparities in acute myeloid leukemia? Cancer Epidemiol Biomark Prev. 2015;24(2):344-9.

9. Borate UM, Mineishi S, Costa LJ. Nonbiological factors affecting survival in younger patients with acute myeloid leukemia. Cancer. 2015;121(21):3877-84.

10. Oran B, Weisdorf DJ. Survival for older patients with acute myeloid leukemia: a population-based study. Haematologica. 2012;97(12):1916-24.

11. Jabo B, Morgan JW, Martinez ME, Ghamsary M, Wieduwilt MJ. Sociodemographic disparities in chemotherapy and hematopoietic cell transplantation utilization among adult acute lymphoblastic and acute myeloid leukemia patients. PLoS One. 2017;12(4):e0174760.

12. Bhatt VR, Chen B, Gyawali B, Lee SJ. Socioeconomic and health system factors associated with lower utilization of hematopoietic cell transplantation in older patients with acute myeloid leukemia. Bone Marrow Transplant. 2018;53(10):1288-94.

13. Bhatt VR, Shostrom V, Gundabolu K, Armitage JO. Utilization of initial chemotherapy for newly diagnosed acute myeloid leukemia in the United States. Blood Advances. 2018;2(11):1277-82.

14. Kristinsson SY, Derolf ÅR, Edgren G, Dickman PW, Björkholm M. Socioeconomic differences in patient survival are increasing for acute myeloid leukemia and multiple myeloma in Sweden. J Clin Oncol. 2009; 27(12):2073-80.

15. Østgård LSG, Nørgaard M, Medeiros BC, Friis LS, Schoellkopf C, Severinsen MT, et al. Effects of education and income on treatment and outcome in patients with acute myeloid leukemia in a tax-supported health care system: a National Population-Based Cohort Study. J Clin Oncol. 2017;35(32): 3678-87.

16. Bertoli S, Tavitian S, Huynh A, Borel C, Guenounou S, Luquet I, et al. Improved outcome for AML patients over the years 2000-2014. Blood Cancer J. 2017;7(12):635

17. Bertoli S, Bérard E, Huguet F, Huynh A, Tavitian S, Vergez F, et al. Time from diagnosis to intensive chemotherapy initiation does not adversely impact the outcome of patients with acute myeloid leukemia. Blood. 2013;121(14): 2618-26.

18. Bories P, Bertoli S, Bérard E, Laurent J, Duchayne E, Sarry A, et al. Intensive chemotherapy, azacitidine, or supportive care in older acute myeloid leukemia patients: an analysis from a regional healthcare network. Am J Hematol. 2014;89(12):E244-E52.

19. Pornet C, Delpierre C, Dejardin O, Grosclaude P, Launay L, Guittet L, et al. Construction of an adaptable European transnational ecological deprivation index: the French version. J Epidemiol Community Health. 2012:66(11):982-9.

20. Townsend P. Deprivation. J Soc Policy. 1987;16(02):125-46. 
21. Charlson ME, Pompei P, Ales KL, MacKenzie CR. A new method of classifying prognostic comorbidity in longitudinal studies: development and validation. J Chronic Dis. 1987;40(5):373-83.

22. Grimwade D, Hills RK, Moorman AV, Walker H, Chatters S, Goldstone AH, et al. Refinement of cytogenetic classification in acute myeloid leukemia: determination of prognostic significance of rare recurring chromosomal abnormalities among 5876 younger adult patients treated in the United Kingdom Medical Research Council trials. Blood. 2010;116(3):354-65.

23. Royston P. Multiple imputation of missing values: further update of ice, with an emphasis on categorical variables. Stata J. 2009;9(3):466.

24. Rubin D. Multiple imputation for nonresponse in surveys; 2008

25. Sterne JAC, White IR, Carlin JB, Spratt M, Royston P, Kenward MG, et al. Multiple imputation for missing data in epidemiological and clinical research: potential and pitfalls. BMJ. 2009;338:b2393.

26. Bryere J, Dejardin O, Launay L, Colonna M, Grosclaude P, Launoy G. Socioeconomic status and site-specific cancer incidence, a Bayesian approach in a French Cancer registries network study. Eur J Cancer Prev. 2018;27(4):391-8.

27. Lamy S, Bettiol C, Grosclaude P, Compaci G, Albertus G, Recher C, et al. The care center influences the management of lymphoma patients in a universal health care system: an observational cohort study. BMC Health Serv Res. 2016;16(a):336.

28. Belot A, Remontet L, Rachet B, Dejardin O, Charvat H, Bara S, et al. Describing the association between socioeconomic inequalities and cancer survival: methodological guidelines and illustration with population-based data. Clin Epidemiol. 2018:10:561-73.

29. Bryere J, Pornet C, Copin N, Launay L, Gusto G, Grosclaude P, et al. Assessment of the ecological bias of seven aggregate social deprivation indices. BMC Public Health. 2017;17(1):86.

\section{Publisher's Note}

Springer Nature remains neutral with regard to jurisdictional claims in published maps and institutional affiliations.

Ready to submit your research? Choose BMC and benefit from:

- fast, convenient online submission

- thorough peer review by experienced researchers in your field

- rapid publication on acceptance

- support for research data, including large and complex data types

- gold Open Access which fosters wider collaboration and increased citations

- maximum visibility for your research: over $100 \mathrm{M}$ website views per year

At $\mathrm{BMC}$, research is always in progress.

Learn more biomedcentral.com/submissions 